\title{
130. A 2D-3D non-contact anthropometric method for daily dressing state-takes young Asian women as example
}

\author{
Ke Ke Sun ${ }^{1}$, Yi Jui Chiu ${ }^{2}$, Yi Cheng Chen ${ }^{3}$, Yu Xiu Wang ${ }^{4}$ \\ ${ }^{1,4}$ School of Art Design and Clothing Engineering, Xiamen University of Technology, Xiamen, China \\ ${ }^{2}$ School of Mechanical and Automotive Engineering, Xiamen University of Technology, Xiamen, China \\ ${ }^{3}$ Graduate Institute of Sports Equipment Technology, University of Taipei, Taipei, Taiwan \\ ${ }^{2}$ Corresponding author \\ E-mail: ${ }^{1}$ sunkeke@xmut.edu.cn, ${ }^{2}$ chiuyijui@xmut.edu.cn, ${ }^{3} y c c h e n @ u t a i p e i . e d u . t w$, \\ 42013110501@xmut.edu.cn
}

Received 23 August 2017; received in revised form 1 September 2017; accepted 2 September 2017

DOI https://doi.org/10.21595/jme.2017.19001

Check for updates

\begin{abstract}
Taking young Asian women as research object, this paper is to develop a 2D-3D non-contact body measurement and calculation method for daily dressing state used to generate the CWH (chest, waist, and hip) circumferences suitable for garment practical applications. The general research approach is 'analyzing related curves of human body, finding their fitting functions and length calculating formulas through mathematical analysis and measuring experiment with large number of samples - extracting effective feature points data in daily dressing state by dynamic and static experiments - calculating such dimensions as width and thickness after curve fitting - generating $\mathrm{CWH}$ circumferences via formulas and making comparison'. The numerical results validate the 2D-3D method presented in this paper as a useful and effective approach. Being different from other 3D non-contact measuring methods, the authors provide a new way to get the CWH measurements in the condition of daily natural dressing, which not only optimizes the non-contact anthropometrics theory, but also breaks the static measuring mode. Furthermore, the bringing forth of the concept of 'daily dressing state' and the laboratory experiments of this paper would be worthy for practical use.
\end{abstract}

Keywords: non-contact anthropometry, 2D-3D, Daily dressing state, dynamic and static experiment, $\mathrm{CWH}$ measurements.

\section{Introduction}

As an important branch of clothing ergonomics, anthropometry is the groundwork ensuring clothing design and manufacturing. In contrast to the traditional contact measuring mode, non-contact ones can offer accurate and full-scale measured data to meet the demands of modern garment digital trend well. 3D scanning and 2D-3D conversion are the main measuring and calculating methods used in the field of non-contact anthropometry, especially the former is well-developed over the past two decades.

For 3D scanning, using laser is popular. Some researchers did related works. For example, Watanabe et al. [1] adopted a laser displacement meter to measure waist circumference and developed the new system for the non-contact measurement of abdominal cross section. Shang et al. [2] used artificial and three-dimensional laser measurement method, through which the sample data were collected in the same situation, to study the national and professional standard size measurement system. In the design of data standards, using statistical methods to analyze the data, and a comprehensive evaluation from two aspects of stability of instrument and data correlation. The practical application was taken as an example to study the tolerance range of standard data. The research works of Wang and Jiang [3] were based on the measurement principle of structured light, a laser rotating body scanning of non-contact measurement system. They gave the requiring of Made to Measure apparel production mode according to individual types and made calibration about body measurement and production in a line. Besides using laser, other researches about the method and application of 3D measuring also carried forward the non-contact measuring 
technique. Such as Zhang et al. [4] developed non-contact measurement of revolving body straightness method. They inspected a $1200 \mathrm{~mm}$ long rotating body with a spatial straightness error of a diameter of $160 \mathrm{~mm}$ in the 3D machine vision metrology. Pan et. al. [5] used C\# and SQL database to design and implement for non contact 3D human body measurement in the human body database management system. Wang et. al. [6] proposed a non-contact 3D body data acquisition method for garment fit evaluation the distribution characteristics of the remaining space between the 3D scanning and clothing. The researches [7-10] used 3D non-contact measurement method to analyze body shape. Remondino [11] explored the generation of 3D models from uncalibrated image sequences. The study process also included the extraction of correspondences on the body using a least squares matching algorithm and the reconstruction of the 3D body model in point cloud form.

Some researches focused on 2D and 2D-3D non-contact measurement, such as Pirre and Shi [12] developed 2D image measurement system, which could determine the size of clothing. They found image-based systems were capable of providing anthropometric measurements, both in terms of accuracy and repeatability, which were better than traditional measurement methods. Yu et al. [13] adopted a wide coverage of optical design. This system could obtain high resolution images in a short distance, and through the computer program into a 3D human body model. Gu et al. [14] developed automatic pattern generation system of men's pants, which is based on two dimensional non-contact human body measurement systems. Guo et al. [15] explored the application of the automatic clipping method in the 2Dimensional non-contact measuring system.

As for the dynamic measurement and application researches, non contact technology of human daily life activity detection include: Kurita [16,17] developed a method for measurement of human motion, which was the electrostatic induction current non-contact type for walking motion detection. Umeda [18] developed of non-contact detection technology, which has been used to measure human vital signs. Deepak et al. [19] and Yutaka et al. [20] utilized 3D non-contact measurement method to discuss knee motion of human body. Kurita [21] developed an effective non-contact technique for the measurement of human stepping. Nicola [22] took advantage of cameras to measure and track moving surfaces of human body parts from multi-image video sequences acquired simultaneously. Yang et al. [23] adopted high speed camera to analyze the landing from different heights, the current research tries to figure out the landing vibration responses to different segments, including the lumbar, knee, hip, neck and the reacting force from ground.

Although the previous works improved the non-contact measuring technique gradually, two of the most common problems or challenges still exist: one is the limitation on the dress of subjects, the other is the static measuring mode. That is, relatively accurate measurements of human body parts can be obtained only when subjects still stand, wearing underwear or straitjacket. In view of garment size designation of the industry, this paper introduces a 2D-3D conversion approach to generate the $\mathrm{CWH}$ measurements in natural daily dressing state, no matter the measuring mode is dynamic or static. Experiments prove that this method, which is free from the dressing constrains and break the static mode, can meet the accuracy requirement. In our approach, through large sample measuring experiment showed in Section 2, laws of the relative curves, such as the silhouette curves of front, back, body side and the cross-section curves of chest, waist, hip of human body, were studied in Section 3, in which their suitable fitting functions and the integral formula of the typical CWH curves were ascertained. Next, in Section 4, on the premise of somatotype, in daily dressing state, static and dynamic experiments were carried to extract the effective feature points' information. Then 2D measurements such as the width and the thickness were measured or calculated out. With the 2D-3D conversional formulas aforementioned, the CWH measurements would be calculated finally. These numerical results and analysis are presented and discussed in Section 5. Conclusion is given in Section 6. The research route is shown in Fig. 1. 


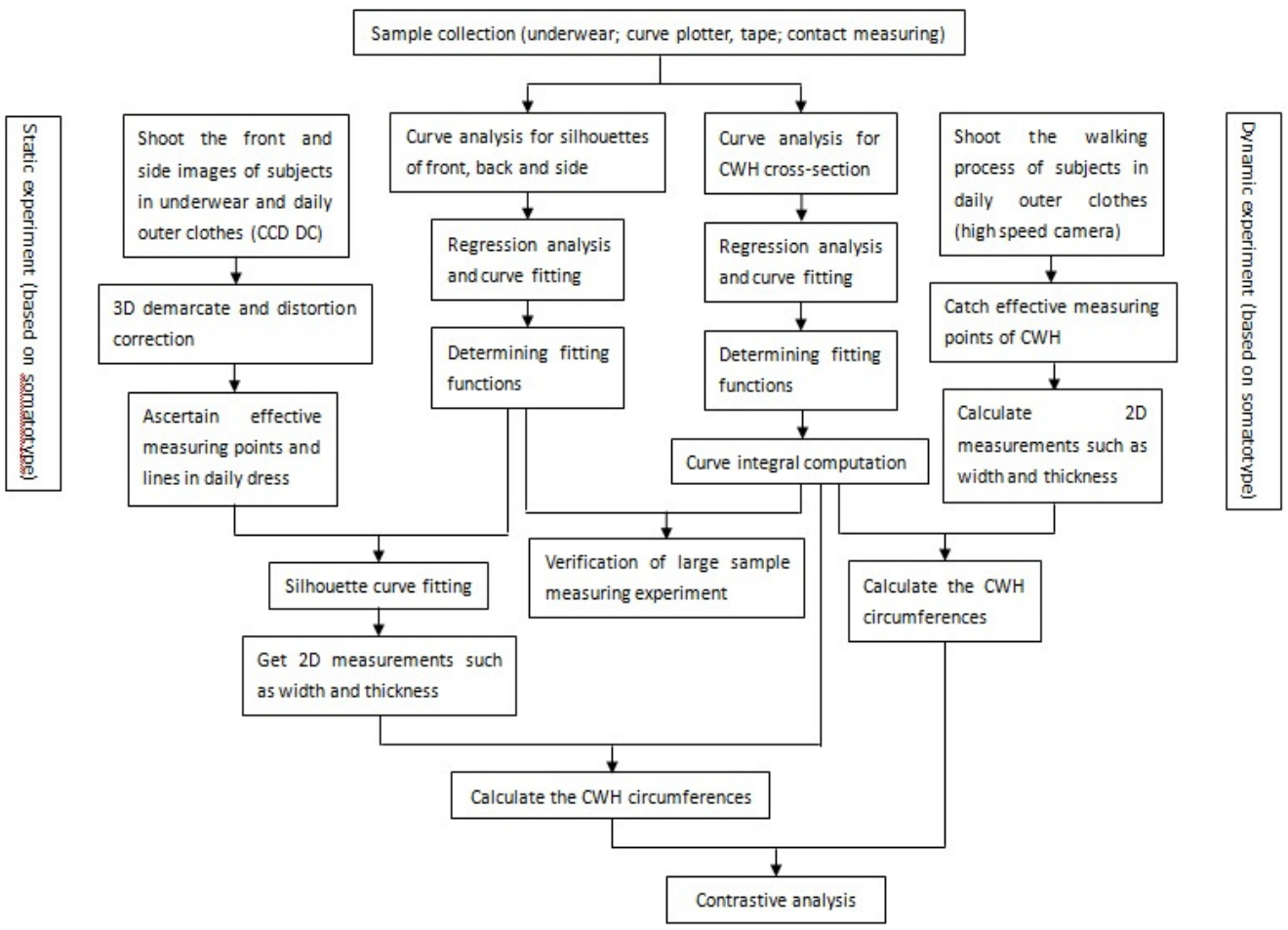

Fig. 1. Research route

\section{Large sample anthropological measuring experiment}

In order to ensure analysis credibility for the curves of silhouette and CWH cross-section, and the relative property between calculating measurements and actual measurements, it is necessary to build a large sample measurement database via traditional contact measuring experiment. In this paper, take Asian young women as research subjects, whose basic requirements are shown in Table 1. The sample size is 81 persons, who are random selected, determined by the known sample data of the earlier study, combining the confidence interval $95 \%$ and the actual experimental condition. The accuracy of CWH measurements is $2 \mathrm{~cm}$, according to the standard of garment size designation. Tape measure, ruler, height measure and curve plotter (Fig. 2) are the main measuring tools. Stature, Chest, Waist, Hip, heights of $\mathrm{CWH}$, widths and depths of CWH, BP to BP, HP to $\mathrm{HP}$, distances of C-W and $\mathrm{W}-\mathrm{H}$, Shoulder are the measuring parts, whose measuring methods refer to GB16160-2008-T. In addition, the cross section curves of CWH and the silhouette curves of front, back, and side of the 81 subjects are plotted by the curve plotter one by one. As the comparison of the later calculating measurements, measuring all the circumferences of $\mathrm{CWH}$ curves manually, the specific results are shown in Table 3.

Table 1. Basic information of subjects

\begin{tabular}{|c|c|}
\hline Age (years) & $18-26$ \\
\hline Height $(\mathrm{cm})$ & $145-175$ \\
\hline Weight $(\mathrm{kg})$ & $40-70$ \\
\hline
\end{tabular}

\section{Relative curves analysis}

Curves associated with the $\mathrm{CWH}$ measuring and calculating include the $\mathrm{CWH}$ cross-section curves and the silhouette curves of the front, back and side. It is not advisable to take any one sample's curves as the analysis basis. Therefore, the typical representative curve figures are 
selected. Take the chest cross-section curve as example. Firstly, divide 81 samples into three types: $0.69-0.77,0.78-0.83$ and $0.84-0.95$, according to the different radius ratios of chest width/depth. Secondly, make smoothing and symmetrical process to the original figures of curves in the same radius ratio range, then overlay them and determine the mean points. Lastly, choose the curve of moderate ratio as the representative chest cross-section curve.

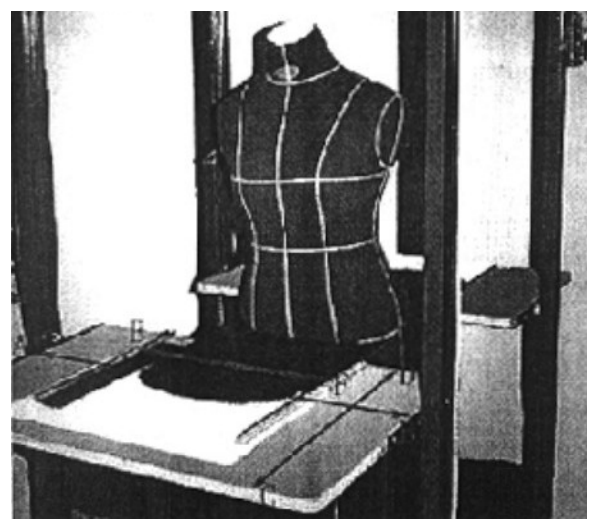

Fig. 2. Curve plotter

\subsection{CWH cross-section curves}

The typical CWH cross-section curves are shown in Fig. 3. For the convenience of analysis and formulation, plane-coordinate system $X O Y$ is set up. For instance, the chest cross-section curve, as shown in Fig. 4, curve above $X$-axis is the front chest curve, below is the back chest curve, segment $W_{1} W_{2}$ is the chest width, $T_{1} T_{2}$ is the chest thickness, $O T_{1}$ is the front chest thickness, $O T_{2}$ is the back one. $O W_{1}=O W_{2}=1 / 2$ chest width, due to the symmetry of human body. Observe the trend of each segment curve, take the segment in $\left(-W_{1}, 0\right)$ as example, a and $\mathrm{b}$ are the demarcation points, for arc $W_{1} a$, the tangent slope is larger, the curvature is less; for arc $\mathrm{ab}$, the tangent slope is decreasing, the curvature is increasing; for $\operatorname{arc} b T_{1}$, the tangent slope is minimum, the curvature is less.

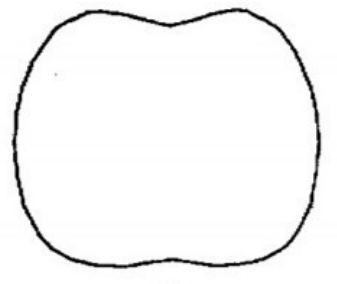

C

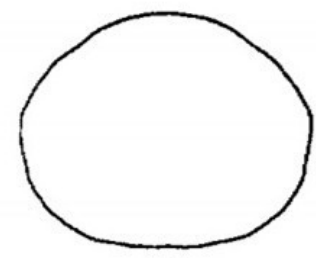

W

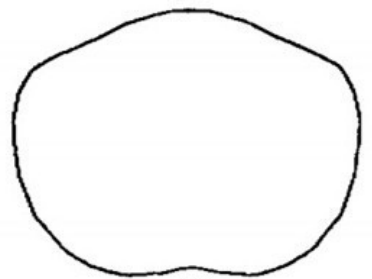

H

Fig. 3. The typical CWH cross-section curves
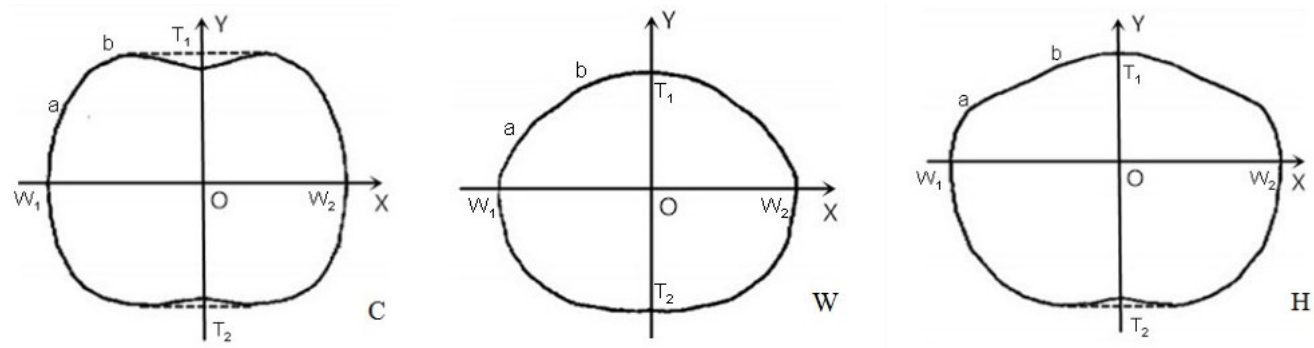

Fig. 4. The typical CWH cross-section curves with $X O Y$ 


\subsubsection{Regression analysis}

Before curve fitting and integrating, getting the thickness proportion of the front and back $\mathrm{CWH}$ curves is the premise. After measuring all the CWH curves obtained in the large sample experiment one by one, the regression formulas is acquired as Eq. (1). It should be noted that there are no difference of the width and thickness measurements after segmenting the CWH curves between using Eq. (1) and human judgment. Therefore, although the fitting confidences $R^{2}$ of the scatter plots are $0.43,0.64,0.51$ respectively, there are no obvious significance to the final calculating results through experimental verification:

$$
\left\{\begin{array}{l}
F C T=C T \times(1-0.5132), \\
B C T=C T \times 0.5132, \\
F W T=W T-(W T \times 0.3483+2.3891), \\
B W T=W T \times 0.3483+2.3891, \\
F H T=H T \times(1-0.6228), \\
B H T=H T \times 0.6228,
\end{array}\right.
$$

where $F$ is front, $B$ is back, $T$ is thickness, $C$ is chest and $W$ is waist.

\subsubsection{Curve fitting}

The existing typical methods of 2D-3D conversion for circumference measurements include super-elliptic curve fitting, EE parametric spline curve fitting, binary linear regression, and quadratic regression. It is found that the first two methods need a mass of statistic data and measuring points, and errors of the last two are larger, which cannot reach the accuracy requirement of this research. According to the principles of fewer measuring points, faster calculating, and higher precision, the logarithmic, trinomial, power, binomial curves are taken as the alternative fitting curves in this paper. Take the front waist curve as example, on which extract feature points evenly, make scatter diagrams of the aforementioned curve fitting, in that order, $R^{2}$ are $0.9884,0.9865,0.9281,0.9052$. After comparing and analyzing the situation of the extracted points and fitting curves, the study found that, for the front and back segments of the $\mathrm{CWH}$ cross-section curve, the confidence of the logarithmic curve fitting is the best, whose shape has the similar trend with the original. Thus, the logarithmic curve is confirmed as the fitting function finally.

Take the 1/2 front waist curve (Fig. 5) as case study, its logarithmic curve equation is given as Eq. (2):

$Y=A * \operatorname{Ln}(x)+B$,

where $A$ is coefficient, $B$ is intercept.

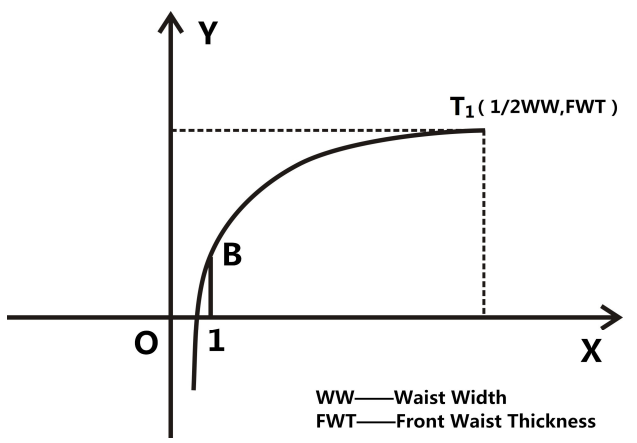

Fig. 5. The abridged general view of $1 / 2$ front waist curve 


\subsubsection{Intercept $B$}

The discussion about the accurate intercept $B$ is given below. Undoubtedly, the logarithmic fitting curve determined by extracting the feature points precisely has the higher fitting precision. Measure the $C W H$ circumferences of 81 samples, calculate the integral computation of their circumferences through changing the intercept B using the successive approximation approach. Make them equal to the known measured circumference, and confirm the intercept $B$, which is regarded as the precise one. The calculation procedure is presented in Fig. 6.

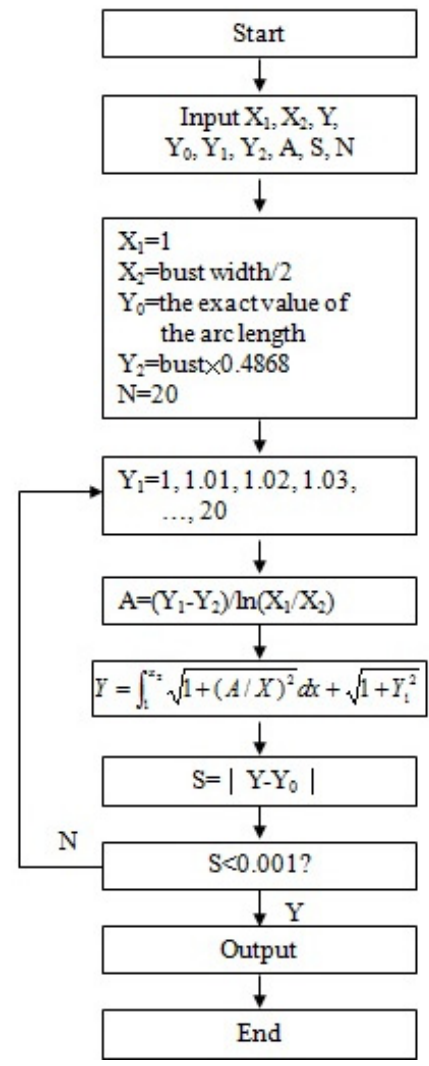

Fig. 6. The calculation procedure of intercept $B$

As for the intercept $B$ obtained using regression analysis, the relative work is described as follows. Through experiment and analysis, infer some kind of corresponding relation among the $C W H$ widths, thickness and the intercept $B$. According to the expression of correlation coefficient (Eq. (3)), the correlation coefficients of the intercept $B$ and the widths, thickness are worked out.

In accordance with the correlation, the $C W H$ intercepts are fitted by multivariable linear regression. To make the data more accurate, the correlation test among the distance of $C-W$, the distance of $W-H$ and the height, and the integral computation using different regression formulas are also conducted. After comparing errors of the integral results, Eq. (4) is written into integral program as this experimental result.

As for the intercept $B$ obtained using regression analysis, the relative work is described as follows. Through experiment and analysis, infer some kind of corresponding relation among the $C W H$ widths, thickness and the intercept $B$. according to the expression of correlation coefficient (Eq. (3)), the correlation coefficients of the intercept $B$ and the widths, thickness are worked out.

In accordance with the correlation, the $C W H$ intercepts are fitted by multivariable linear 
regression. To make the data more accurate, the correlation test among the distance of $C$ - $W$, the distance of $W-H$ and the height, and the integral computation using different regression formulas are also conducted. After comparing errors of the integral results, Eq. (4) is written into integral program as this experimental result:

$\rho_{X Y}=\frac{\operatorname{Cov}(X, Y)}{\sqrt{D(X)} \sqrt{D(Y)}}$,

where $\operatorname{Cov}(X, Y)$ is co-variation of $X$ and $Y, D(X), D(Y)$ are variances of $X$ and $Y$.

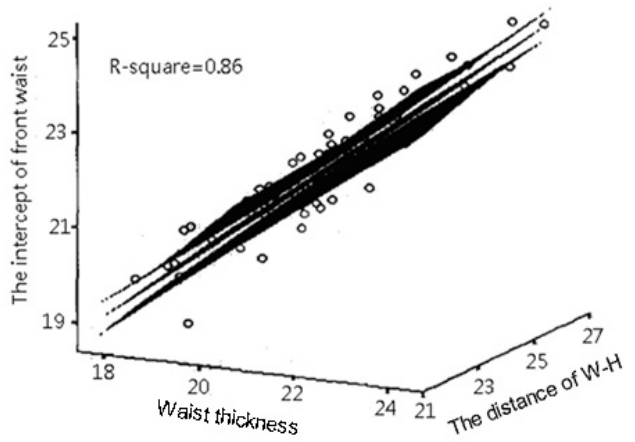

a)

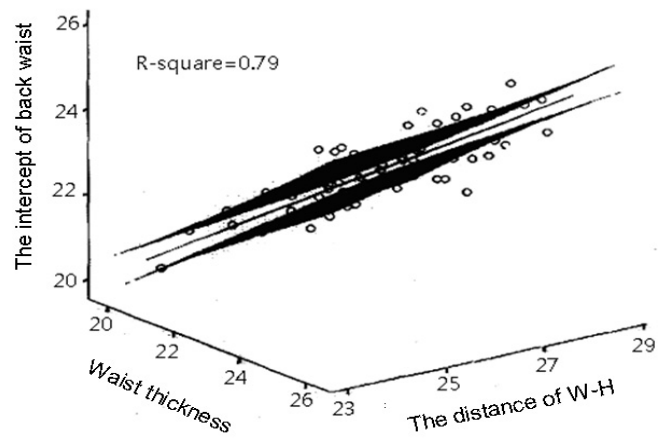

b)

Fig. 7. The scatter diagrams and responsive plane of the intercepts of the front and back waist

\section{In Fig. 7:}

The Intercept of $F W=0.82+0.11 \times W T+0.05 \times$ the distence of $W-H$,

The Intercept of $B W=1.44+0.25 \times W T-0.06 \times$ the distence of $W-H$,

The Intercept of $F C=5.06-0.31 \times C W+0.47 \times C T$,

The Intercept of $B C=7.93-0.20 \times C W+0.22 \times C T$,

The Intercept of $F H=0.145+0.056 \times H W+0.039 \times H T+0.056 \times$ the distence of $W-H$,

The Intercept of $B H=4.315-0.235 \times H W+0.397 \times H T+0.115 \times$ the distence of $W-H$,

where $H$ is hip.

Take the intercepts of the front and back waist as instance, Fig. 7 shows its scatter diagrams and responsive plane.

\subsubsection{Circumference calculation of the fitted curves}

On the basis of Eq. (2), the girth of the fitted curve is expressed as Eq. (5). The effective integral range is $(1,1 / 2$ width), let $k=1 / 2$ width, that is Eq. (6). The curvature of the curve in the range of $(0,1)$ is very small, and it cannot be extracted enough information and fitted in MATLAB, as a result, this paper employs the calculation method of hypotenuse (Eq. (7)) to obtain its approximate length. Through a large number of experimental verification, the error of this algorithm is minor:

$$
\begin{aligned}
& y=\int \sqrt{1+(a / x)^{2}} d x, \\
& y=\int_{1}^{k} \sqrt{1+(a / x)^{2}} d x, \\
& a \hat{c} \approx \sqrt{a c^{\prime 2}+c c^{\prime 2}} .
\end{aligned}
$$




\subsection{Silhouette curves}

The analysis process and methods of this section is similar to the CWH cross-section curves presented in section 3.1. Here not repeat them because of the limited space. By research, the fitting functions of the silhouette curves of the front, back and side respectively are $\operatorname{cscvn}()$, Spline Interplant and $\operatorname{cscvn}()$. Fig. 8 shows their fitting comparison, in which the red is the typical body silhouette curves, and the blue is the fitted curves.

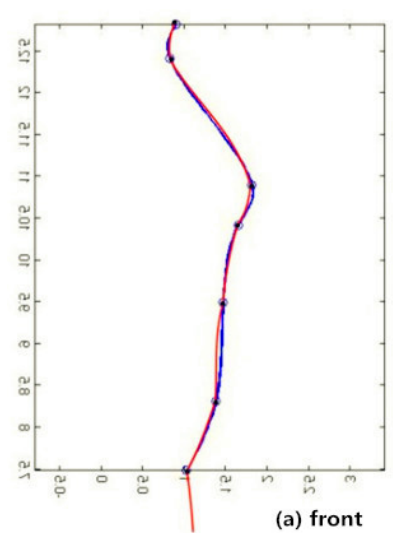

(a) front

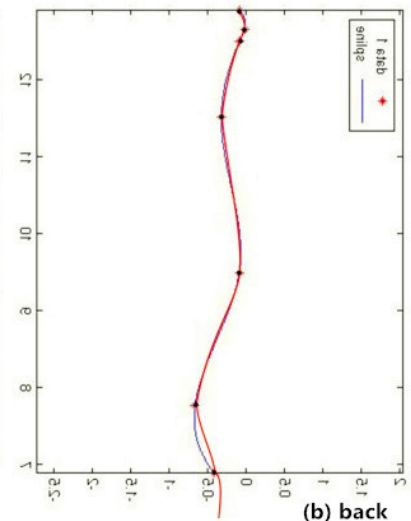

(b) back

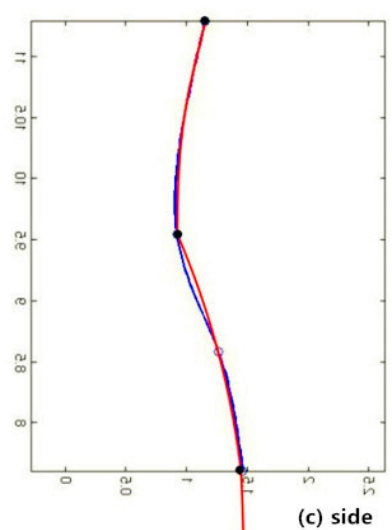

(c) side

Fig. 8. Fitting comparison

\section{Static and dynamic experiment in daily dressing state}

The basic information of subjects in these two experiments is as follows:

- age: 18-26;

- somatotype: thin, intermediate, plump;

- dressing types: blouse + trousers (jeans) of spring and autumn, T-shirt + tailored skirt (A-line skirt) of summer; sweater + jeans (pants) of winter.

Table 2. Somatotype criterion

\begin{tabular}{|c|c|c|c|}
\hline \multirow{2}{*}{ Index } & \multicolumn{3}{|c|}{ Somatotype } \\
\cline { 2 - 4 } & Thin & Normal & Plump \\
\hline TNF & $<32$ & $32-40$ & $>40$ \\
\hline
\end{tabular}

Among them, the somatotype base is the body linear density index TNF, whose calculation is shown as Eq. (8). Table 2 shows the classification criterion. The reason why choose TNF is that TNF reflect the figure type feature of the Asian young people more better than the other universal methods such as girth difference, Rorel index, Verveck index and Bust/Height. In the base of somatotype and research of the previous large sample experiment, only three typical figure subjects are selected for case study in this section. The detailed steps are described as below:

$T N F=(W / L) * 100$,

where $W$ is weight $(\mathrm{kg}) ; L$ is height $(\mathrm{cm})$.

\subsection{Static experiment}

Main equipment: three dimensional calibration frame, fixed tripod, OLYMPUS C2500L digital camera, image capture card and a computer.

Indoor environment: room temperature $26^{\circ} \mathrm{C}$; ordinary fluorescent light.

Experimental procedures: 1) Shoot the frontal and side digital images of each subject in 
sequence. In which, when shoot the front, the subject is required to do up hair, stand on the mark in the calibration frame, place feet shoulder width apart, raise arms whose angle with the body side is about $40^{\circ}$. Unlike the above, when shoot the side, the subject stand on the other mark, and her arms cling to the body without occluding the silhouette curves of the front and back. 2) Three dimensional calibration and distortion correction, to effectively reduce the measuring error caused by optical imaging. 3) Measure the related heights, lengths, and $\mathrm{CWH}$ widths and thicknesses. Fig. 9 shows the experimental diagrammatic sketch.
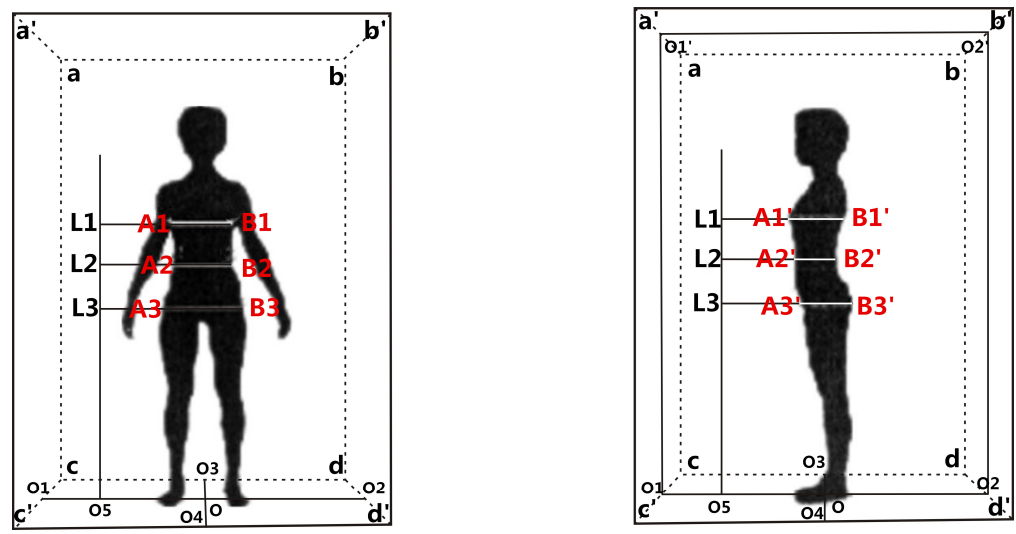

Fig. 9. The static experiment arrangement

\subsection{Dynamic experiment}

Main hardware and software: 3D real-time motion analysis system, Raptor-E infrared high-speed camera, witch balls whose diameters are $1 \mathrm{~cm}$, Cortex 3.0, and computer. Indoor environment is the same.

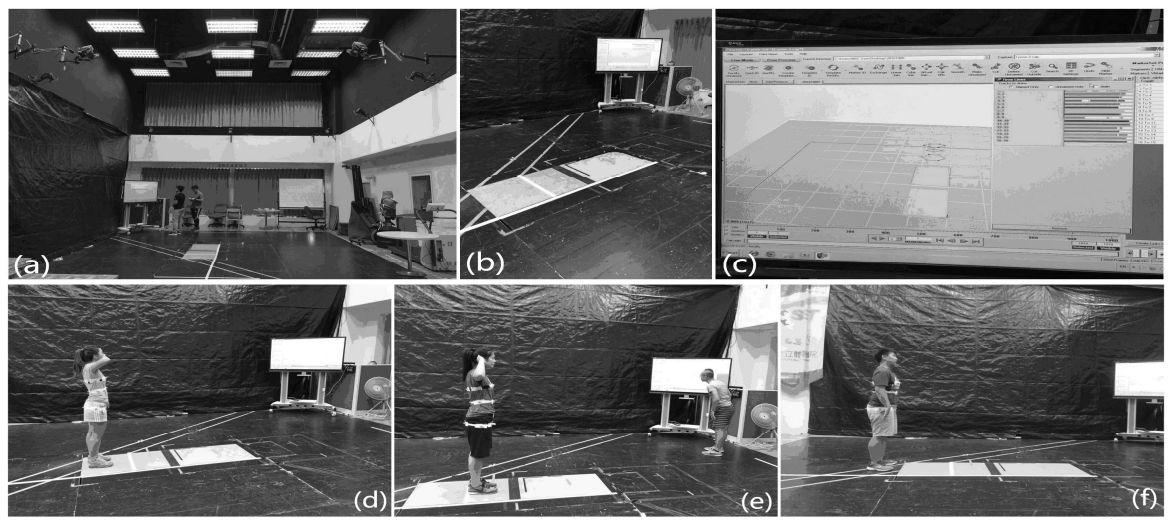

Fig. 10. The dynamic experiment arrangement

Experimental procedures: 1) Bind elastic bands and paste witch balls on the BWH locations of the subjects in natural wearing state. 2) Test the range of capturing motion and debug the system. 3) Ask the subject walk as usual in the tested space. 4) The dynamic data of the witch balls are generated in the software system Cortex 3.0.

The experimental arrangement and scene are shown in Fig. 10(a) shows the site arrangement; (b) shows the walking area; (c) shows the system which reflects the dynamic situation of the featured points; (d), (e) and (f) show the subjects of three different figure types. Note: In dynamic experiment, influence of different dresses to the capturing process is less, owing to the binding band, thus only select the summer wearing model as the experimental wearing. 


\section{Numerical results and analysis}

The results comparison between the calculating circumferences and measuring circumferences in the large sample experiment are shown in Table 3-Table 5.

Table 3. Results of chest measurement of the large sample experiment

\begin{tabular}{|c|c|c|c|c|c|c|c|}
\hline Sample No. & Calculating $C$ & Measuring $C$ & Error & Sample No. & Calculating $C$ & Measuring $C$ & Error \\
\hline 75 & 80.83 & 82.30 & -1.47 & 70 & 76.90 & 76.90 & 0.00 \\
\hline 39 & 80.44 & 81.90 & -1.46 & 19 & 78.61 & 78.60 & 0.01 \\
\hline 55 & 79.65 & 81.10 & -1.45 & 60 & 86.11 & 86.10 & 0.01 \\
\hline 76 & 75.66 & 77.10 & -1.44 & 10 & 79.28 & 79.20 & 0.08 \\
\hline 66 & 78.84 & 80.20 & -1.36 & 23 & 81.73 & 81.60 & 0.13 \\
\hline 61 & 83.24 & 84.60 & -1.36 & 25 & 79.93 & 79.80 & 0.13 \\
\hline 30 & 80.75 & 82.10 & -1.35 & 81 & 82.64 & 82.50 & 0.14 \\
\hline 27 & 81.78 & 83.10 & -1.32 & 72 & 79.76 & 79.60 & 0.16 \\
\hline 56 & 87.43 & 88.70 & -1.27 & 74 & 87.36 & 87.10 & 0.26 \\
\hline 37 & 82.06 & 83.30 & -1.24 & 53 & 77.37 & 77.10 & 0.27 \\
\hline 52 & 82.26 & 83.40 & -1.14 & 15 & 78.51 & 78.20 & 0.31 \\
\hline 43 & 77.24 & 78.20 & -0.96 & 16 & 83.22 & 82.90 & 0.32 \\
\hline 18 & 74.15 & 75.10 & -0.95 & 41 & 78.68 & 78.30 & 0.38 \\
\hline 45 & 72.85 & 73.80 & -0.95 & 69 & 73.30 & 72.90 & 0.40 \\
\hline 80 & 80.40 & 81.30 & -0.90 & 54 & 74.21 & 73.80 & 0.41 \\
\hline 35 & 82.76 & 83.60 & -0.84 & 9 & 80.92 & 80.50 & 0.42 \\
\hline 50 & 84.89 & 85.70 & -0.81 & 77 & 83.03 & 82.60 & 0.43 \\
\hline 12 & 78.17 & 78.90 & -0.73 & 5 & 84.43 & 84.00 & 0.43 \\
\hline 34 & 89.14 & 89.85 & -0.71 & 29 & 82.55 & 82.10 & 0.45 \\
\hline 57 & 82.57 & 83.20 & -0.63 & 20 & 76.10 & 75.60 & 0.50 \\
\hline 48 & 79.08 & 79.70 & -0.62 & 51 & 86.04 & 85.50 & 0.54 \\
\hline 44 & 82.66 & 83.20 & -0.54 & 47 & 80.69 & 80.10 & 0.59 \\
\hline 13 & 76.69 & 77.20 & -0.51 & 8 & 78.66 & 77.90 & 0.76 \\
\hline 24 & 78.93 & 79.40 & -0.47 & 63 & 82.79 & 82.00 & 0.79 \\
\hline 73 & 77.85 & 78.30 & -0.45 & 68 & 79.60 & 78.80 & 0.80 \\
\hline 7 & 78.96 & 79.40 & -0.44 & 28 & 82.51 & 81.60 & 0.91 \\
\hline 67 & 77.76 & 78.20 & -0.44 & 17 & 79.62 & 78.70 & 0.92 \\
\hline 6 & 78.21 & 78.60 & -0.39 & 49 & 83.56 & 82.60 & 0.96 \\
\hline 14 & 83.41 & 83.70 & -0.29 & 3 & 79.78 & 78.80 & 0.98 \\
\hline 58 & 83.99 & 84.20 & -0.21 & 59 & 81.01 & 80.00 & 1.01 \\
\hline 11 & 77.72 & 77.90 & -0.18 & 32 & 83.72 & 82.70 & 1.02 \\
\hline 65 & 78.63 & 78.80 & -0.17 & 33 & 84.07 & 83.00 & 1.07 \\
\hline 78 & 80.75 & 80.90 & -0.15 & 31 & 81.77 & 80.70 & 1.07 \\
\hline 79 & 85.20 & 85.35 & -0.15 & 62 & 74.67 & 73.60 & 1.07 \\
\hline 46 & 76.97 & 77.10 & -0.13 & 40 & 83.08 & 82.00 & 1.08 \\
\hline 71 & 74.69 & 74.80 & -0.11 & 22 & 78.61 & 77.40 & 1.21 \\
\hline 42 & 78.80 & 78.90 & -0.10 & 26 & 81.74 & 80.50 & 1.24 \\
\hline 36 & 84.44 & 84.50 & -0.06 & 4 & 78.75 & 77.50 & 1.25 \\
\hline 64 & 80.78 & 80.80 & -0.02 & 2 & 80.50 & 79.20 & 1.30 \\
\hline 1 & 78.19 & 78.20 & -0.01 & 38 & 77.64 & 76.20 & 1.44 \\
\hline 21 & 77.50 & 77.50 & 0.00 & & & & \\
\hline
\end{tabular}

Table 3-5 show that, the absolute error of calculating $\mathrm{CWH}$ are respectively in $(-1.47 \mathrm{~cm} \sim 1.44 \mathrm{~cm}), \quad(-1.50 \mathrm{~cm} \sim 1.51 \mathrm{~cm}), \quad(-1.60 \mathrm{~cm} \sim 1.63 \mathrm{~cm})$, which basically meet the requirement of $\pm 1.5 \mathrm{~cm}$ in garment industry. The errors caused by the measuring and calculating process are improved as follows. Overlap and piece the 4-5 groups of curve figures of the largest positive and negative error and the minimal absolute error, as Fig. 11 shown. It can be seen that, 
curves of the lager positive error- $\mathrm{P}$ model, the negative error- $\mathrm{N}$ model and the smaller absolute error- $\mathrm{S}$ model have the similar traits in shape and trend. Classify CWH curves in the above crosssection shapes in advance, using image recognition. Calculate the intercept $B$ with different regression formulas for different types of curves, and then run the integrations. This process can decrease the error effectively.

Table 4. Results of waist measurement of the large sample experiment

\begin{tabular}{|c|c|c|c|c|c|c|c|}
\hline Sample no. & Calculating $W$ & Measuring $W$ & Error & Sample no. & Calculating $W$ & Measuring $W$ & Error \\
\hline 58 & 72.10 & 73.6 & -1.50 & 1 & 67.50 & 67.7 & -0.20 \\
\hline 74 & 70.18 & 71.6 & -1.42 & 33 & 68.34 & 68.5 & -0.16 \\
\hline 14 & 66.41 & 67.8 & -1.39 & 22 & 62.85 & 63 & -0.15 \\
\hline 44 & 62.22 & 63.5 & -1.28 & 80 & 69.66 & 69.8 & -0.14 \\
\hline 75 & 66.00 & 67.2 & -1.20 & 36 & 69.81 & 69.9 & -0.09 \\
\hline 68 & 63.23 & 64.4 & -1.17 & 34 & 74.91 & 75 & -0.09 \\
\hline 67 & 59.54 & 60.7 & -1.16 & 7 & 62.24 & 62.3 & -0.06 \\
\hline 17 & 60.50 & 61.6 & -1.10 & 53 & 64.05 & 64.1 & -0.05 \\
\hline 28 & 66.89 & 67.8 & -0.91 & 52 & 68.77 & 68.8 & -0.03 \\
\hline 18 & 58.36 & 59.2 & -0.84 & 57 & 63.60 & 63.6 & 0.00 \\
\hline 72 & 62.87 & 63.7 & -0.83 & 48 & 62.12 & 62.1 & 0.02 \\
\hline 45 & 59.58 & 60.4 & -0.82 & 70 & 57.92 & 57.9 & 0.02 \\
\hline 41 & 58.70 & 59.5 & -0.80 & 21 & 60.05 & 60 & 0.05 \\
\hline 78 & 66.90 & 67.7 & -0.80 & 51 & 72.09 & 72 & 0.09 \\
\hline 65 & 62.04 & 62.8 & -0.76 & 13 & 61.60 & 61.5 & 0.10 \\
\hline 26 & 60.59 & 61.3 & -0.71 & 30 & 62.95 & 62.8 & 0.15 \\
\hline 54 & 56.19 & 56.9 & -0.71 & 40 & 65.50 & 65.3 & 0.20 \\
\hline 71 & 60.30 & 61 & -0.70 & 39 & 65.01 & 64.8 & 0.21 \\
\hline 31 & 67.06 & 67.7 & -0.64 & 19 & 64.91 & 64.7 & 0.21 \\
\hline 62 & 58.98 & 59.5 & -0.62 & 63 & 66.81 & 66.6 & 0.21 \\
\hline 9 & 66.59 & 67.2 & -0.61 & 61 & 69.39 & 69.1 & 0.29 \\
\hline 25 & 66.24 & 66.8 & -0.56 & 49 & 68.30 & 68 & 0.30 \\
\hline 5 & 69.07 & 69.6 & -0.53 & 32 & 74.27 & 73.9 & 0.37 \\
\hline 64 & 62.37 & 62.9 & -0.53 & 66 & 59.82 & 59.45 & 0.37 \\
\hline 35 & 66.98 & 67.5 & -0.52 & 81 & 67.25 & 66.8 & 0.45 \\
\hline 23 & 65.88 & 66.4 & -0.52 & 38 & 62.58 & 62.1 & 0.48 \\
\hline 20 & 61.28 & 61.8 & -0.52 & 46 & 60.89 & 60.4 & 0.49 \\
\hline 69 & 59.09 & 59.6 & -0.51 & 79 & 69.30 & 68.8 & 0.50 \\
\hline 75 & 69.30 & 69.8 & -0.50 & 24 & 61.21 & 60.7 & 0.51 \\
\hline 37 & 68.75 & 69.2 & -0.45 & 8 & 62.12 & 61.55 & 0.57 \\
\hline 2 & 61.45 & 61.9 & -0.45 & 59 & 62.10 & 61.5 & 0.60 \\
\hline 60 & 70.16 & 70.6 & -0.44 & 55 & 67.31 & 66.7 & 0.61 \\
\hline 27 & 64.49 & 64.9 & -0.41 & 11 & 59.63 & 59 & 0.63 \\
\hline 56 & 70.80 & 71.2 & -0.40 & 47 & 70.12 & 69 & 1.12 \\
\hline 29 & 66.61 & 67 & -0.39 & 10 & 60.23 & 59 & 1.23 \\
\hline 15 & 64.76 & 65.1 & -0.34 & 3 & 64.94 & 63.7 & 1.24 \\
\hline 16 & 68.26 & 68.6 & -0.34 & 6 & 62.06 & 60.8 & 1.26 \\
\hline 73 & 61.48 & 61.8 & -0.32 & 4 & 63.11 & 61.8 & 1.31 \\
\hline 42 & 66.33 & 66.6 & -0.27 & 50 & 69.03 & 67.6 & 1.43 \\
\hline 77 & 68.27 & 68.5 & -0.23 & 12 & 67.71 & 66.2 & 1.51 \\
\hline 43 & 65.88 & 66.1 & -0.22 & & & & \\
\hline
\end{tabular}

The results and the comparisons between the calculating circumference s and the measuring circumference $\mathrm{s}$ of the static and dynamic experiments, in which all the subjects wear different daily clothes and their body shape were classified in advance, are shown in Table 6 and Table 7.

Table 6 shows that, different dressings have some impacts on the selection of the feature points 
and the circumference calculation of the fitted curves. When the subjects wear clothes of soft textured and elastic fabric, whose styles highlight the body curves and fit, the errors of the measuring experiment are less.

Table 5. Results of hip measurement of the large sample experiment

\begin{tabular}{|c|c|c|c|c|c|c|c|}
\hline Sample no. & Calculating $H$ & Measuring $H$ & Error & Sample no. & Calculating $H$ & Measuring $H$ & Error \\
\hline 36 & 97.50 & 99.1 & -1.60 & 30 & 88.33 & 88.3 & 0.03 \\
\hline 78 & 88.59 & 90.1 & -1.51 & 77 & 93.14 & 93.1 & 0.04 \\
\hline 74 & 92.32 & 93.8 & \begin{tabular}{|l|}
-1.48 \\
\end{tabular} & 14 & 89.06 & 89 & 0.06 \\
\hline 29 & 87.14 & 88.6 & -1.46 & 27 & 85.48 & 85.4 & 0.08 \\
\hline 44 & 88.59 & 90 & -1.41 & 17 & 85.93 & 85.8 & 0.13 \\
\hline 12 & 84.87 & 86.2 & \begin{tabular}{|l|}
-1.33 \\
\end{tabular} & 55 & 91.93 & 91.8 & 0.13 \\
\hline 40 & 90.08 & 91.4 & -1.32 & 19 & 86.93 & 86.8 & 0.13 \\
\hline 34 & 91.38 & 92.7 & -1.32 & 79 & 95.07 & 94.9 & 0.17 \\
\hline 7 & 86.91 & 88.15 & -1.24 & 37 & 87.11 & 86.9 & 0.21 \\
\hline 21 & 81.87 & 83.1 & -1.23 & 22 & 86.82 & 86.6 & 0.22 \\
\hline 45 & 79.39 & 80.6 & -1.21 & 52 & 91.62 & 91.4 & 0.22 \\
\hline 58 & 91.81 & 93 & -1.19 & 59 & 84.52 & 84.3 & 0.22 \\
\hline 18 & 82.25 & 83.4 & -1.15 & 20 & 82.28 & 82 & 0.28 \\
\hline 41 & 85.88 & 87 & -1.12 & 5 & 95.02 & 94.7 & 0.32 \\
\hline 16 & 89.52 & 90.6 & -1.08 & 3 & 83.32 & 83 & 0.32 \\
\hline 31 & 89.73 & 90.7 & -0.97 & 69 & 79.73 & 79.4 & 0.33 \\
\hline 32 & 90.93 & 91.9 & -0.97 & 75 & 91.33 & 91 & 0.33 \\
\hline 15 & 89.26 & 90.2 & -0.94 & 68 & 88.24 & 87.9 & 0.34 \\
\hline 57 & 84.97 & 85.9 & -0.93 & 11 & 83.79 & 83.4 & 0.39 \\
\hline 8 & 82.05 & 82.9 & -0.85 & 56 & 90.62 & 90.2 & 0.42 \\
\hline 13 & 86.40 & 87.1 & -0.70 & 66 & 87.89 & 87.4 & 0.49 \\
\hline 24 & 85.47 & 86.1 & -0.63 & 72 & 84.89 & 84.4 & 0.49 \\
\hline 9 & 90.71 & 91.3 & -0.59 & 43 & 91.50 & 91 & 0.50 \\
\hline 64 & 85.34 & 85.9 & -0.56 & 60 & 90.93 & 90.4 & 0.53 \\
\hline 2 & 84.56 & 85.1 & -0.54 & 80 & 87.35 & 86.8 & 0.55 \\
\hline 38 & 84.00 & 84.45 & -0.45 & 49 & 91.85 & 91.3 & 0.55 \\
\hline 67 & 82.35 & 82.7 & -0.35 & 61 & 88.75 & 88.2 & 0.55 \\
\hline 46 & 81.28 & 81.6 & -0.32 & 33 & 89.35 & 88.7 & 0.65 \\
\hline 42 & 92.42 & 92.7 & -0.28 & 63 & 91.26 & 90.6 & 0.66 \\
\hline 6 & 84.63 & 84.9 & -0.27 & 39 & 84.27 & 83.6 & 0.67 \\
\hline 47 & 90.31 & 90.5 & -0.19 & 10 & 84.87 & 84.2 & 0.67 \\
\hline 73 & 80.27 & 80.4 & -0.13 & 4 & 80.74 & 80 & 0.74 \\
\hline 28 & 89.68 & 89.8 & -0.12 & 54 & 80.33 & 79.3 & 1.03 \\
\hline 62 & 80.69 & 80.8 & -0.11 & 50 & 88.90 & 87.8 & 1.10 \\
\hline 71 & 82.31 & 82.4 & -0.09 & 76 & 88.32 & 87.1 & 1.22 \\
\hline 51 & 85.41 & 85.5 & -0.09 & 25 & 88.45 & 87.2 & 1.25 \\
\hline 70 & 80.92 & 81 & -0.08 & 35 & 93.77 & 92.5 & 1.27 \\
\hline 26 & 86.94 & 87 & -0.06 & 23 & 90.06 & 88.5 & 1.56 \\
\hline 1 & 85.85 & 85.9 & -0.05 & 81 & 86.71 & 85.1 & 1.61 \\
\hline 65 & 79.10 & 79.1 & 0.00 & 53 & 86.63 & 85 & 1.63 \\
\hline 48 & 84.82 & 84.8 & 0.02 & & & & \\
\hline
\end{tabular}

Table 7 shows that, although the information of the featured points captured from the subjects' walking state is different, the $C W H$ calculated vales are less different, which can meet the industry requirement. Certainly, the function of binding bands is not ignored, but as the feasibility discussion of the method, this experiment provides a possibility. And it should be studied how to get rid of the $C W H$ binding bands and obtain the featured points' data in the future practical application. 
Table 6. Results of the static experiment

\begin{tabular}{|c|c|c|c|c|}
\hline \multicolumn{5}{|c|}{ Subject A (thin) } \\
\hline Dressing $C W H$ & Elastic underwear & Blouse +A-line skirt & T-shirt +A-line skirt & Sweater + jeans \\
\hline$C$ & 79.92 & 83.64 & 82.28 & 81.14 \\
\hline$W$ & 61.92 & 64 & 63.78 & 62.44 \\
\hline$H$ & 83 & 80.68 & 82.46 & 83.76 \\
\hline \multicolumn{5}{|c|}{ Subject B (normal) } \\
\hline Dressing $C W H$ & Elastic underwear & Blouse + trousers & T-shirt + tailored skirt & Sweater + pants \\
\hline$C$ & 83.90 & 88 & 84.70 & 88.50 \\
\hline$W$ & 66.14 & 70.52 & 68.50 & 70.84 \\
\hline$H$ & 88.48 & 91.92 & 90.46 & 90.82 \\
\hline \multicolumn{5}{|c|}{ Subject C (plump) } \\
\hline Dressing $C W H$ & Elastic underwear & Blouse + jeans & T-shirt +A-line skirt & Sweater + jeans \\
\hline$C$ & 93.02 & 95.26 & 93.08 & 91.58 \\
\hline$W$ & 70.78 & 78.22 & 72.34 & 70.32 \\
\hline$H$ & 99.14 & 100.24 & 100.1 & 101.2 \\
\hline
\end{tabular}

Table 7. Results of the dynamic experiment

\begin{tabular}{|c|c|c|c|c|c|c|c|}
\hline \multirow{2}{*}{$B$ somatotype } & \multicolumn{5}{|c|}{ Calculated values $(\mathrm{cm})$} & \multirow{2}{*}{ Actual $(\mathrm{cm})$} & \multirow{2}{*}{ Error range $(\mathrm{cm})$} \\
\hline & 1 & 2 & 3 & 4 & 5 & & \\
\hline Thin & 80.83 & 80.54 & 80.90 & 80.45 & - & 80.1 & $0.35 \sim 0.80$ \\
\hline Normal & 85.22 & - & 84.74 & 84.35 & 84.75 & 84.9 & $-0.55 \sim 0.22$ \\
\hline Plump & 96.77 & 96.53 & 96.40 & 97.63 & 96.70 & 96.0 & $0.40 \sim 1.63$ \\
\hline \multirow{2}{*}{$W$ somatotype } & \multicolumn{5}{|c|}{ Calculated values $(\mathrm{cm})$} & \multirow{2}{*}{ Actual $(\mathrm{cm})$} & \multirow{2}{*}{ Error range $(\mathrm{cm})$} \\
\hline & 1 & 2 & 3 & 4 & 5 & & \\
\hline Thin & 62.05 & 61.91 & 62.11 & 62.13 & - & 61.2 & $0.71 \sim 0.93$ \\
\hline Normal & 70.42 & - & 69.79 & 70.25 & 70.49 & 69.0 & $0.79 \sim 1.49$ \\
\hline Plump & 84.69 & 85.04 & 85.92 & 85.71 & 85.92 & 85.0 & $-0.31 \sim 0.92$ \\
\hline \multirow{2}{*}{$H$ somatotype } & \multicolumn{5}{|c|}{ Calculated values $(\mathrm{cm})$} & \multirow{2}{*}{ Actual $(\mathrm{cm})$} & \multirow{2}{*}{ Error range $(\mathrm{cm})$} \\
\hline & 1 & 2 & 3 & 4 & 5 & & \\
\hline Thin & 87.37 & 87.60 & 87.04 & 87.36 & - & 87.5 & $-0.46 \sim 0.10$ \\
\hline Normal & 99.79 & - & 100.18 & 100.29 & 99.94 & 100.5 & $-0.71 \sim-0.21$ \\
\hline Plump & 104.01 & 104.66 & 104.39 & 104.86 & 105.02 & 105.4 & $-1.39 \sim-0.38$ \\
\hline
\end{tabular}

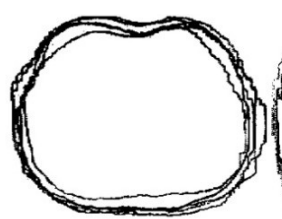

C-P

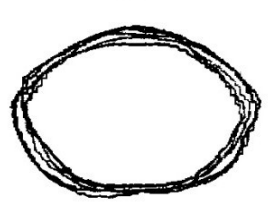

W-P

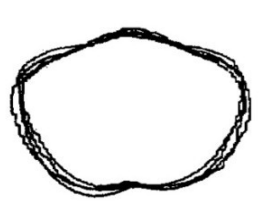

$\mathrm{H}-\mathrm{P}$

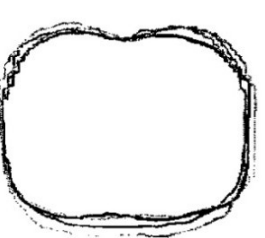

C-S

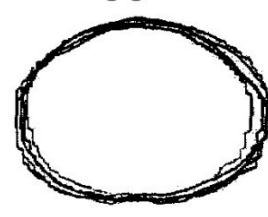

W-S

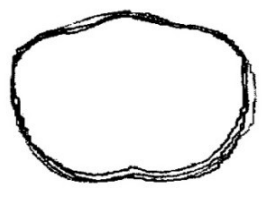

$\mathrm{H}-\mathrm{S}$

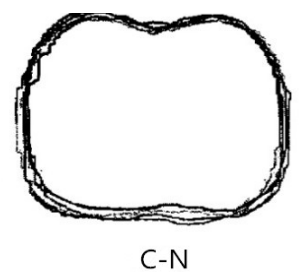

$\mathrm{C}-\mathrm{N}$

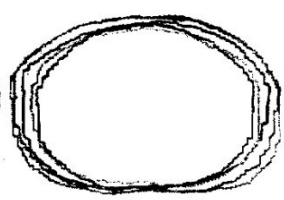

W-N

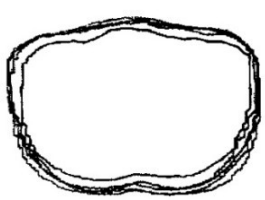

$\mathrm{H}-\mathrm{N}$

Fig. 11. Actual CWH cross-section curves of different error type 


\section{Conclusions}

Comparing the length dimension which can be obtained more easily, how to generate the $\mathrm{CWH}$ girth using limited feature points is one of the main subjects in the field of 2D-3D non-contact anthropometry used for garment industry. Taking Asian young women as researching objects, by regression analysis and curve fitting, algorithms of the $C W H$ circumferences were presented in this paper. As for the daily dressing state and the dynamic measuring mode, representative experiments were conducted, which had guidance meaning for making non-contact body measurement more convenient. By extracting the feature points in natural wearing state, static and dynamic, and using the algorithms mentioned above, the $C W H$ measurements were well obtained. This process is effective and provides a foundation for the rapid shape recognition and classification.

\section{Acknowledgements}

All the research works are supported by Fujian Social Science Project (FJ2016C118), Fujian Nature Foundation (2016J01039), Xiamen University of Technology Talent Foundation (YKJ15027R), Xiamen City Project (3502Z20173037).

\section{References}

[1] Watanabe A., Seno S. I., Kobayashi H., Kato S., Shimazu H. Development of non-contact body cross-section measurement system and evaluation for the practical use. Transactions of Japanese Society for Medical and Biological Engineering, Vol. 49, Issue 1, 2011, p. 156-162.

[2] Shang X. M., Chen J., Wang Z. Y. Stability and Correlation analysis of data obtained by contact and non-contact measuring body. Advanced Materials Research, Vol. 156, Issue 157, 2011, p. 600-606.

[3] Wang Y., Jiang S. S. The laser rotating of non-contact body scanning system. Journal of Fiber Bioengineering and Informatics, Vol. 8, Issue 1, 2015, p. 91-103.

[4] Zhang S. X., Liu Y. L., Liu W. L., Ran D. G., Wang J. C. Non-contact measurement of revolving body straightness. Proceedings of the Second International Symposium on Instrumentation Science and Technology, 2002, p. 132-136.

[5] Pan L., Yao T., Yao C., Wang J. Stability and correlation analysis of data obtained by contact and non-contact measuring body. Advanced Materials Research, Vol. 1048, 2014, p. 545-549.

[6] Wang Z., Zhong Y. Q., Chen K. J., Ruan J. Y., Zhu J. C. 3D human body data acquisition and fit evaluation of clothing. Advanced Materials Research, Vol. 989, Issue 994, 2014, p. 4161-4164.

[7] Zhang X., Wang Y. Y., Ran L. H., Feng A. L., He K. T., Liu T. J., Niu J. W. Human dimensions of Chinese minors. Lecture Notes in Computer Science, Vol. 6777, 2011, p. 37-45.

[8] Yang M., Guo P. P., Gu B. F., Liu G. L. Analysis of body shape differences of young males in south and north China. Textile Bioengineering and Informatics Symposium Proceedings, 2014, p. 891-898.

[9] Wu Z. Z., Liao S. S., Nie L., Wang Z., Wang C. H., Zhou S. H. Design and implementation of human size measurement system based on images. Journal of Hunan University Natural Sciences, Vol. 37, Issue 9, 2010, p. 88-92.

[10] Gu B. F., Liu G. L. Automatic modelling of the lower bodies of young females based on digital photographs. Journal of Fiber Bioengineering and Informatics, Vol. 7, Issue 2, 2014, p. 247-260.

[11] Remondino F. 3-D reconstruction of static human body shape from image sequence. Computer Vision and Image Understanding, Vol. 93, 2004, p. 65-85.

[12] Pirre M., Shi Y. Performance of a 2D image-based anthropometric measurement and clothing sizing system. Applied Ergonomics, Vol. 31, 2000, p. 445-451.

[13] Yu W., Ng R., Yan S. CubiCam - a new approach of 3D body scanning system. Journal of HongKong Polytechnic University, Vol. 28, 2002, p. 55-62.

[14] Gu B. F., Kong H. Y., Gu P. Y., Su J. Q., Liu G. L. Study of 2D non-contact anthropometric system and application. Proceedings of International Conference on Future Computer Sciences and Application, 2011, p. 150-153.

[15] Guo P. P., Liu G. L., Dai H. Q. The application of automatic clipping in 2D non-contact measuring system. Textile Bioengineering and Informatics Symposium Proceedings, 2015, p. 589-595. 
[16] Kurita K. Novel non-contact and non-attached technique for detecting sports motion. Measurement: Journal of the International Measurement Confederation, Vol. 44, Issue 8, 2011, p. 1361-1366.

[17] Kurita K. Human physical activity measurement method based on electrostatic induction. 11th IMEKO TC14 Symposium on Laser Metrology for Precision Measurement and Inspection in Industry, 2014, p. 82-85.

[18] Umeda T., Takagi R., Jyo K. Non-contact monitoring system for body pose and respiration with radar sensor. 52nd Annual Conference of the Society of Instrument and Control Engineers of Japan, 2013, p. 2372-2373.

[19] Deepak S., Tavakoli M., Atkinson P., Anseth S., Raley T., Walter N. External knee geometry surface variation as a function of subject anthropometry and flexion angle for human and surrogate subjects. SAE Technical Papers, 2007.

[20] Yutaka A., Akinori S., Hiroshi H., Sho Y., Yasuhiro O., Hideki M. A method for non-contact measurement of knee load in sport motion. IEEE, 2007, p. 2724-2729.

[21] Kurita K. Development of non-contact measurement system of human stepping. SICE Annual Conference, 2008, p. 1067-1070.

[22] Nicola D. A. Surface measurement and tracking of human body parts from multi-image video sequences. ISPRS Journal of Photogrammetry and Remote Sensing, Vol. 56, 2002, p. 360-375.

[23] Yang C. H., Lin P. C., Chiu Y. J. The vibration response analysis of segments in human body landing. International Symposium on Sports Biomechanics, 2007, p. 199-200. 